\title{
Shoulder external/internal rotation peak torques ratio side-asymmetry, mean work and power ratios balance worsening due to different fatigue resistance of the rotator muscles in male handball players
}

Inese Pontaga

Department of Anatomy, Physiology, Biochemistry and Hygiene of Latvian Academy of Sports

Education, Riga, Latvia

Corrensponding author:

Inese Pontaga

Department of Anatomy, Physiology,

Biochemistry and Hygiene of Latvian Academy of

Sports Education

Brivibas Street 333

Riga, LV-1006, Latvia

E-mail: Inese.Pontaga@Ispa.Iv

\section{Summary}

Introduction: Fatigue could worsen shoulder cuff rotator balance if fatigue of the external rotation (ER) could appear faster in comparison with the internal rotation (IR). The shoulder rotation peak torques ( $\left.\tau_{\max }\right)$ side-asymmetry and muscle endurance effect on the mean work (W), mean power $(P)$ and their ratio of ER/IR in handball players was investigated.

Methods: Eighteen semi-professional male handball players participated. The tests were performed using an isokinetic dynamometer by the shoulder isokinetic internal-external rotation movements at angular velocities of $60 \% \mathrm{~s}, 240 \% \mathrm{~s}$ via concentric contractions. The $\mathrm{W}, \mathrm{P}$ were determined and their ratios of the ER/IR were calculated for the first ten and last ten repetitions of the movements at $240 \%$ s.

Results: Muscle side asymmetry in $\mathrm{T}_{\max }, \mathrm{W}, \mathrm{P}$ was not observed at $240 \%$ s. The W, P of the ER and ratios of the ER/IR ( $\mathrm{W}_{\mathrm{ER}} / \mathrm{W}_{\mathrm{IR}}=0.76$ (dominant $\mathrm{D}$ ), 0.74 (non-dominant $\mathrm{N}$ ) arm; $\mathrm{P}_{\mathrm{ER}} / \mathrm{P}_{\mathrm{IR}}=0.68 \mathrm{D}, 0.70 \mathrm{~N}$ ) in the last ten repetitions were significantly lower

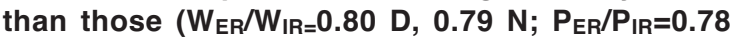
$\mathrm{D}, 0.77 \mathrm{~N}$ ) in the first ten movements.

Conclusion: Fatigue resistance of the ER was lower than that of the IR. Therefore, the ER/IR ra- tios of $\mathrm{W}, \mathrm{P}$ decrease due to repeated rotation movements.

Level of evidence: Illa.

KEY WORDS: fatigue, glenohumeral stability, handball, isokinetic dynamometry, shoulder rotation sideasymmetry, strength endurance.

\section{Introduction}

Repetitive throwing observed in athletes trained in overhead sports can contribute to increase in strength and power of the shoulder internal rotation (IR); however, the increase in strength and power of the external rotation (ER) is not proportional ${ }^{1-3}$. Peak torque ratio of shoulder ER/IR in athletes trained in sports with overhead throwing movements, such as water polo ${ }^{2}$, tennis and baseball players ${ }^{1}$ varied from 0.55 to 0.68 . This ratio is specific to sports specialization because throwing movement variations depend on the sport. For athletes trained in sports with regular overhead activities, the peak torque ratios of the ER/IR commonly decrease in the dominant shoulder but do not change in the non-dominant shoulder ${ }^{4}$. Codine et al. ${ }^{1}$ observed significantly lower ratios in dominant arms compared to non-dominant arms in nonathletes, runners and baseball players.

This significant side asymmetry of the peak torque ratio of the ER/IR was not observed in water polo players $^{2}$, baseball pitchers ${ }^{4}$, tennis players ${ }^{1}$, or adult ${ }^{5}$ and adolescent $^{6}$ team handball players.

Balance between the shoulder internal and external rotation strength is needed not only for appropriate throwing mechanics but also for glenohumeral joint stability providing. Non-balanced action between internal and external rotation of the shoulder is considered a risk factor of shoulder injury in athletes trained in sports with regular overhead movements of $\mathrm{arms}^{7}$. The ER strength and power are especially important in the arm deceleration stage during the throwing motion ${ }^{8}$.

Fatigue could worsen non-balanced action of shoulder rotation if fatigue of the ER could appear faster in comparison with the IR. This might be an additional shoulder injury risk factor in the athlete trained in sports with overhead arms motions due to alteration of overhead movement kinematics. For example, Chen et al. ${ }^{9}$ determined the humeral head significant 
superior migration in all positions of the arm abduction due to fatiguing exercises series of deltoid and rotator cuff muscles. Teyhen et al. ${ }^{10}$ also observed increase of superior migration of the humeral head after rotator cuff muscle fatigue caused by special dynamic arm elevation exercise protocols in healthy males. Joshi et al. ${ }^{11}$ revealed that shoulder ER muscle fatigue exercise protocol performed by overhead athletes caused increased scapular upward rotation range of motion during a functional throwing motion task and shoulder joint range of motion reduction in joint extension and internal rotation. They detected an increase in infraspinatus muscle activation by electromyography, which might predispose the infraspinatus muscle to injury through chronically increased activation $^{11}$. Maenhout et al. ${ }^{12}$ detected that fatiguing protocol (the upper extremity external-internal rotation movement exercise with the shoulder abducted to the angle of $90^{\circ}$ repetitions to achieve low-quality motion) caused acromiohumeral distance increase, upward and external rotation, and posteriorly tilted position of scapula in the abducted upper extremity in recreational overhead athletes. This position changes corresponded with a protective impingement-sparing situation and could be explained by the scapula compensating for glenohumeral shoulder muscle fatigue.

Fatigue resistance of the shoulder rotator cuff muscles is especially important in team's sport athletes because of the repetitive nature of the throwing motions. Therefore, the fatigue of the shoulder muscles can decrease throwing performance and kinematics due to lower strength and power production, worsen proprioceptive sense of the joints involved in the throwing motion, change the shoulder ER/IR torque, work and power ratios due to differential fatigue resistance of the muscle groups, and can be a reason for shoulder joint injury, especially at the end of a playmatch $^{11,13}$. For example, handball players must coordinate their specific movements of passing, catching, throwing, checking and blocking during the entire game (two thirty minutes periods) ${ }^{14,15}$. Therefore, the performance of handball players depends on the peak torque and power of the shoulder muscles, but the fatigue resistance of the shoulder rotator cuff muscles is especially important in maintaining throwing performance to the end of the handball playmatch.

Mullaney et al. ${ }^{16}$ and Nocera et al. ${ }^{17}$ determined no significant decline of the shoulder IR and ER maximal isometric strength (one repetition maximum - 1MR) and of the isokinetic peak torque following pitching or throwing in athletes. Their results prove that isometric assessment is not appropriate testing mode to evaluate functions of shoulder rotator muscles because these muscles predominantly function dynamically during throwing motion ${ }^{16,17}$.

Andrade et al. ${ }^{18}$ investigated influence of simulated handball game activities (100 steps and 20 arm throws to a goal) on the peak torque of IR and ER, their ratio $(E R / I R)$ and on ball throwing performance in elite adult male players. Unlike data of Mullaney et al. ${ }^{16}$ and Nocera et al. ${ }^{17}$, Andrade et al. ${ }^{18}$ determined significant decrease of IR more than of ER peak torque after these activities but ER/IR peak torque ratio did not changed. The simulated game activities were insufficient to impair the ball throwing performance. The mean heart rate during simulated game activities was 153 (SD 13) beats per minute. This confirms that the intensity of simulated handball game activities was low in comparison with real handball play-match with a mean heart rate 160-170 beats/ min., close to the anaerobic threshold ${ }^{15}$. A total running distance per play-match varied from 3.9 to $4.7 \mathrm{~km}^{14,15}$, but in the investigation of Andrade et al. ${ }^{18}$ it was only 100 steps. Therefore, the simulated handball game activities performed by Andrade et al. ${ }^{18}$ are not comparable with the real intensity of handball play-match activities.

Using of the shoulder rotator muscles peak torques decrease as indicator of fatigue is doubtful because only in the investigation of Andrade et al. ${ }^{18}$ significant diminishing of IR and ER peak torques is observed; other Authors ${ }^{16,17}$ did not observed significant changes of the rotation peak torques due to fatigue of shoulder muscles. The fatigue resistance difference in shoulder IR and ER and changes in ER/IR mean work and power ratios due to isokinetic internal-external rotation exercises in handball players has not been investigated. Therefore, investigation of shoulder rotator muscle fatigue resistance using 20 repetitions of isokinetic internal-external rotation exercises at the fast velocity of movements in laboratory could give insight into real difference in strength endurance of IR and ER comparable with fatigue resistance of these muscles during handball play-match.

The aim of the investigation was to determine the shoulder rotation peak torques $\left(T_{\max }\right)$ side-asymmetry and muscle endurance effect on the mean work (W), mean power $(P)$ and their ratio of external/internal rotation in handball players.

\section{Materials and methods}

Eighteen semi-professional male athletes who played in Premium league teams were informed of the possible risks of participation and voluntarily participated in the investigation (every athlete signed an informed consent form to take part in the investigation). Their training experience in team handball ranged from seven to ten years. These athletes trained five times per week (one and half to two hours per day) and played regularly on the weekends. The study was performed in accordance with the standards of the Ethics Committee of the local Council of Sciences, in accordance with the Declaration of Helsinki and the ethical standards of the Journal ${ }^{19}$. All shoulder joints of the athletes did not underwent to previous surgery, were injury free and painless during the investigation. The athletes' height was measured using an Ultrasound Height Measuring Unit MZ10020 (ADE, Hamburg). Body mass was measured in handball players 
Shoulder external/internal rotation peak torques ratio side-asymmetry, mean work and power ratios balance worsening due to different fatigue resistance of the rotator muscles in male handball players

wearing briefs using a Body Composition Analyser BC-418 (Tanita Corporation, Japan), and the error of measurement was $0.1 \mathrm{~kg}$.

The mean age, body height, body mass and body mass index of the tested athletes were 22.0 (SD 2.0) years, 188 (SD 6) cm, 88.0 (SD 9.0) kg and 25.1 (SD 2.2) $\mathrm{kg} / \mathrm{m}^{2}$, respectively.

The tests were performed using the dynamometer system REV-9000 (Technogym, Gambettola, Italy). Hadzic et al. ${ }^{20}$ proved the excellent test-retest reliability and validity of the shoulder internal and external rotation measurements using an isokinetic dynamometer REV 9000 (Technogym SpA, Gambettola, Forli, Italy) for concentric and isometric peak torque values in the short range of movement. The isokinetic dynamometer was calibrated according to manufacturer's specifications and checked before to testing of every participant.

Every semi-professional handball player was tested only once in preseason period. Repeated measurements were not performed because the athletes were not available for the second test in our study.

Before the isokinetic test, the athletes performed a general warm-up for 15 minutes. The warm-up included specific active stretching exercises for shoulder, arm and hand muscles. Passive internal-external rotation motions in the shoulder joint were performed for $90 \mathrm{~s}$ at an angular velocity of $120 \%$ s just before the investigation. The athletes were given detailed verbal instructions of the procedures and performed five submaximal warm-up repetitions before the test.

\section{The test protocol}

The athlete was seated on a bench with an elbow resting on an input shaft. A dynamometer level arm was adjusted to the length of the athlete's forearm. The elbow was flexed to $90^{\circ}$. The humerus was abducted at a right angle $\left(90^{\circ}\right)$ to the trunk. The internal and external rotation movements were performed in the scapular plane, which lies parallel to the scapula plane surface. Greenfield et al. ${ }^{21}$ determined that testing in the scapular plane was preferable because the shoulder ER strength values in this plane were significantly higher than those in the frontal plane.

The range of movement was from $20^{\circ}$ of the shoulder ER to $100^{\circ}$ of the IR $\left(0^{\circ}\right.$ coincided with the vertical axis of the ROM). The ROM was selected as the maximal shoulder external-internal rotation range, which did not cause any discomfort in athletes during movements (and to diminish appearance of the delayed onset muscle soreness in the days following the test). The test ROM was chosen to be maximally safe for semi-professional team handball players with the aim to not affect their throwing performance. This ROM was limited by the mechanical rotational axis of the equipment that was possible only in one plane (isolated internal-external rotation without any other movements in the shoulder joint) and stabilization of the trunk.

The chest and pelvis were stabilized using straps to exclude trunk movement. The handball player's feet were placed on a support platform. The measure- ments were corrected for the effects of gravity. The shoulder isokinetic concentric internal and external rotation movements were tested at angular velocity values of $60 \%$ and $240 \%$ s. Internal and external rotation movements were repeated five times at the velocity of $60 \%$ s and 20 times at the velocity of $240 \%$ s. Participants were verbally encouraged to maximally move the extremity "as hard and as fast as possible" during concentric testing. The testing order of the dominant and non-dominant arms was randomized. The passive internal-external rotation motions in the shoulder joint were performed for $90 \mathrm{~s}$ at the angular velocity of $120 \%$ s before the test and between tests of different velocities for better recovery and relaxation of involved muscles, tendons and ligaments before and after maximal active concentric contractions.

\section{Statistical analysis}

The data distribution was evaluated from the values of the skewness and kurtosis and skewness and kurtosis t-test analysis. The data on height, body mass, athlete age, peak torque, mean work and mean power of the shoulder rotator muscles were normally distributed in the dominant and non-dominant arms. Therefore, using parametric statistics is appropriate.

Peak torque $\left(T_{\max }\right)$ values $(\mathrm{N} \cdot \mathrm{m})$ of the IR and $E R$ were obtained from the best repetition (greatest peak torque). The $\mathrm{W}$ and $\mathrm{P}$ of the IR and ER of the dominant and non-dominant arms were determined for the first ten and last ten repetitions of shoulder rotation movements at an angular velocity of $240 \%$ s. Ellenbecker and Roetert 22 used a muscular fatigue protocol consisting of 20 maximal-effort concentric contractions of shoulder ER and IR at $300 \%$ s. They calculated a relative fatigue ratio by dividing the work in the last 10 repetitions by the work in the first 10 repetitions. We calculated the mean work and power ratios of the shoulder ER/IR for the first ten and last ten repetitions of movements. Mean values and standard deviations for all characteristics were calculated.

Student's dependent t-test for paired data groups was employed to determine differences between the mean $T_{\max }$ values and their ratios of the shoulder rotation in the dominant and non-dominant arms, and between the mean $W$, mean $P$, and mean $W$ and $P$ ratios of the ER/IR in the first ten and last ten repetitions of the shoulder internal-external rotation at the velocity of $240 \%$ s. The differences were considered significant at $p<0.05$. Microsoft Excel 2007 was used to perform all statistical procedures.

\section{Results}

The $T_{\max }$ of the dominant shoulder IR was significantly higher in comparison with the $T_{\max }$ of the non-dominant shoulder IR at the slow velocity of $60 \% / s(p=0.03)$, Table I. The $T_{\max }$ of the ER and the shoulder ER/IR peak torque ratio did not differed significantly in the dominant and non-dominant shoulder at the velocity of $60 \%$ s. A 
Table I. The peak torques $\left(T_{\max }\right)$ and peak torque ratios (ER/IR) of shoulder internal (IR) and external rotation (ER) at slow $(60 \%)$ and fast $(240 \%)$ velocity of movement.

\begin{tabular}{|c|c|c|c|c|c|c|}
\hline \multirow{2}{*}{$\begin{array}{l}\text { Velocity of } \\
\text { movem. } \\
\text { Arm }\end{array}$} & \multicolumn{3}{|l|}{$60 \% \mathrm{~s}$} & \multicolumn{3}{|l|}{$240 \% \mathrm{~s}$} \\
\hline & $\mathrm{T}_{\max } \mathrm{IR}(\mathrm{N} \cdot \mathrm{m})$ & $\mathrm{T}_{\max } \mathrm{ER}(\mathrm{N} \cdot \mathrm{m})$ & $\mathrm{T}_{\max } \mathrm{ER} / \mathrm{T}_{\max } \mathrm{IR}$ & $\mathrm{T}_{\max } \mathrm{IR}(\mathrm{N} \cdot \mathrm{m})$ & $\mathrm{T}_{\max } \mathrm{ER}(\mathrm{N} \cdot \mathrm{m})$ & $\begin{array}{l}\mathrm{T}_{\max } \mathrm{ER} / \mathrm{T}_{\max } \\
\mathrm{IR}\end{array}$ \\
\hline Dominant & $\begin{array}{l}77 \\
(\mathrm{SD} 16)\end{array}$ & $\begin{array}{l}59 \\
\text { (SD 13) }\end{array}$ & $\begin{array}{l}0.78 \\
\text { (SD 0.16) }\end{array}$ & $\begin{array}{l}58 \\
\text { (SD 10) }\end{array}$ & $\begin{array}{l}42 \\
\text { (SD 12) }\end{array}$ & $\begin{array}{l}0.73 \\
\text { (SD 0.18) }\end{array}$ \\
\hline Non-dom. & $\begin{array}{l}71 \\
\text { (SD 17) }\end{array}$ & $\begin{array}{l}57 \\
\text { (SD 13) }\end{array}$ & $\begin{array}{l}0.82 \\
(S D 0.11)\end{array}$ & $\begin{array}{l}52 \\
\text { (SD 9) }\end{array}$ & $\begin{array}{l}38 \\
\text { (SD 9) }\end{array}$ & $\begin{array}{l}0.73 \\
(S D 0.13)\end{array}$ \\
\hline $\begin{array}{l}\text { Sign. of } \\
\text { difference }\end{array}$ & $\begin{array}{l}p=0.03 \\
\text { Significant }\end{array}$ & $\begin{array}{l}p=0.16 \\
\text { N.S. }\end{array}$ & $\begin{array}{l}p=0.14 \\
\text { N.S. }\end{array}$ & $\begin{array}{l}p=0.07 \\
\text { N.S. }\end{array}$ & $\begin{array}{l}p=0.06 \\
\text { N.S. }\end{array}$ & $\begin{array}{l}p=0.44 \\
\text { N.S. }\end{array}$ \\
\hline
\end{tabular}

N.S., not significant.

statistically significant side-asymmetry in the $T_{\max }$ of the shoulder rotation in the dominant and non-dominant arms in semi-professional male handball players at the high angular velocity of $240 \%$ s was not observed (Tab. I). The $T_{\max }$ of shoulder IR and ER was higher at slow movements $(60 \%)$ than at fast movements $(240 \% \mathrm{~s})$. The $T_{\max }$ ratio of ER/IR decreased with the increase of the angular velocity of movement.

Mean $W$ and $P$ values of the shoulder IR in the first ten and last ten shoulder internal-external rotation movements did not differ significantly. The values of the ER in the last ten movement repetitions were significantly lower than those of the first ten shoulder external rotation movements (Tab. II).

The mean $W$ and mean $P$ ratios of the shoulder ER/IR were significantly lower in the last ten rotation movements compared to the first ten internal-external rotation motions at the velocity of $240 \%$ s (Tab. II).

Table II. The mean W and mean P values of shoulder internal (IR) and external rotation (ER) and their ratio values of shoulder ER/IR in the first ten and last ten repetitions of the shoulder internal-external rotation movements at the velocity of $240 \%$ s.

\begin{tabular}{|c|c|c|c|c|c|c|}
\hline \multirow{2}{*}{$\begin{array}{l}\text { Arm } \\
\text { Repetitions }\end{array}$} & \multicolumn{3}{|l|}{ Dominant } & \multicolumn{3}{|c|}{ Non-dominant } \\
\hline & First ten & Last ten & Sign. of diff. & First ten & Last ten & Sign. of diff. \\
\hline $\mathrm{W}_{\mathrm{IR}}(\mathrm{J})$ & $\begin{array}{l}68 \\
(S D 14)\end{array}$ & $\begin{array}{l}65 \\
\text { (SD 14) }\end{array}$ & $p=0.09$ & $\begin{array}{l}61 \\
\text { (SD 12) }\end{array}$ & $\begin{array}{l}58 \\
\text { (SD 13) }\end{array}$ & $p=0.08$ \\
\hline$P_{\mathrm{IR}}(\mathrm{W})$ & $\begin{array}{l}118 \\
\text { (SD 25) }\end{array}$ & $\begin{array}{l}119 \\
\text { (SD 26) }\end{array}$ & $p=0.36$ & $\begin{array}{l}103 \\
\text { (SD 24) }\end{array}$ & $\begin{array}{l}100 \\
\text { (SD 22) }\end{array}$ & $p=0.21$ \\
\hline$W_{E R}(J)$ & $\begin{array}{l}54 \\
\text { (SD 14) }\end{array}$ & $\begin{array}{l}49 \\
\text { (SD 11) }\end{array}$ & $p=0.01$ & $\begin{array}{l}48 \\
\text { (SD 12) }\end{array}$ & $\begin{array}{l}43 \\
\text { (SD 12) }\end{array}$ & $p=0.01$ \\
\hline$P_{E R}(W)$ & $\begin{array}{l}91 \\
\text { (SD 24) }\end{array}$ & $\begin{array}{l}79 \\
\text { (SD 19) }\end{array}$ & $p=0.01$ & $\begin{array}{l}79 \\
\text { (SD 21) }\end{array}$ & $\begin{array}{l}71 \\
\text { (SD 20) }\end{array}$ & $p=0.01$ \\
\hline $\mathrm{W}_{\mathrm{ER}} / \mathrm{W}_{\mathrm{IR}}$ & $\begin{array}{l}0.80 \\
(S D 0.16)\end{array}$ & $\begin{array}{l}0.76 \\
\text { (SD 0.16) }\end{array}$ & $p=0.02$ & $\begin{array}{l}0.79 \\
(S D 0.10)\end{array}$ & $\begin{array}{l}0.74 \\
(S D 0.08)\end{array}$ & $p=0.02$ \\
\hline$P_{E R} / P_{I R}$ & $\begin{array}{l}0.78 \\
\text { (SD 0.16) }\end{array}$ & $\begin{array}{l}0.68 \\
\text { (SD 0.16) }\end{array}$ & $p=0.01$ & $\begin{array}{l}0.77 \\
\text { (SD 0.14) }\end{array}$ & $\begin{array}{l}0.70 \\
\text { (SD 0.14) }\end{array}$ & $p=0.01$ \\
\hline
\end{tabular}


Shoulder external/internal rotation peak torques ratio side-asymmetry, mean work and power ratios balance worsening due to different fatigue resistance of the rotator muscles in male handball players

\section{Discussion}

A side asymmetry in the peak torques, mean work and mean power of shoulder rotation at the fast velocity of $240 \%$ s was not observed in semi-professional male team handball players $(p=0.06-0.44)$, Table I. This gives evidence about proportional development of both shoulders' rotator muscles due to training in handball. A significant side asymmetry was determined only in the peak torque of shoulder IR at the slow angular velocity of $60 \%$ with higher value in the dominant shoulder in comparison with the non-dominant $(p=0.03)$. The peak torques, mean work and power ratios of the shoulder ER/IR varied from 0.73 to 0.82 before fatigue (Tabs. I, II). The mean work and mean power of shoulder ER and the mean work and mean power ratios of ER/IR (from 0.68 to 0.76 ) in the last ten repetitions of shoulder internal-external rotation movements were significantly lower than those in the first ten repetitions ( $p=0.010-0.024)$, Table II. These results proved the lower fatigue resistance of the shoulder ER compared to the IR in isokinetic concentric exercises.

Strength of the study: the group of participants was homogeneous, they all were elite level semi-professional handball players. The measurements were performed with high accuracy due to using of isokinetic dynamometry and application of standard protocol of fatigue development during isokinetic exercises.

Weakness of the study: the shoulder ER muscle fatigue was caused by isokinetic shoulder internal-external rotation exercises only in the concentric contractions of the muscles in the present investigation of handball players. Therefore, the results in real handball play-match can differ from these data. Shoulder external-internal rotation motion is very fast (close to $6000 \%$ s) and is an important component of throwing. The ROM in real throwing movement is close to $180^{\circ}$ with a large external rotation angle $\left(85^{\circ}\right.$ from the vertical axis $)^{8}$. Increased external rotation allows storage of elastic energy in eccentric contractions of ER. The eccentric action of ER in the shoulder internal rotation extreme positions decelerates the arm. The ROM in the present study was from $20^{\circ}$ of the shoulder ER to $100^{\circ}$ of the IR $\left(0^{\circ}\right.$ coincided with the vertical axis of the ROM): only $80^{\circ}$. The test ROM was chosen to be maximally safe for handball players with the aim to not affect their throwing performance. This ROM was limited by the mechanical rotational axis of the equipment that was only in one plane (isolated internal-external rotation) and stabilization of the trunk. The ROM used in our tests is approximately twice smaller than in a real throwing motion. The rotation appears together with horizontal abduction-adduction, flexion-extension in shoulder joint and the trunk extension-flexion in real throwing motion ${ }^{8,23}$. This rotation is not possible to perform in isokinetic tests with a stabilized joint moving only in one plane. The ROM used in our test coincides with the middle part and the part of internal rotation with mostly concentric contractions of the rotator muscles but without extreme positions of the real throwing motion with eccentric action of the muscles.

Team handball players use both arms in ball passing and sometimes throw using their non-dominant arm. Therefore, the $\mathrm{T}_{\max }$ and mean $\mathrm{W}$ and $\mathrm{P}$ side asymmetry of shoulder rotation was not observed in our semiprofessional handball players, excepting the higher $\mathrm{T}_{\max }$ of the dominant shoulder IR at the slow velocity of $60 \%$ s.

There are four different throwing techniques in handball: the standing throw with run-up, jump throw, pivot throw and diving throw ${ }^{23}$. Each throw involves different proportions of shoulder muscle contributions to achieve high ball speed, and different proportions of the concentric and eccentric contractions may be used in the shoulder range of movements during throwing motions. Therefore, the peak torque ratios of shoulder ER/IR at rest were higher in our handball players (from 0.73 to 0.82 ) than in other throwing athletes $(E R / I R \text { ratios varied from } 0.55 \text { to } 0.68)^{1,2}$. We suggest that the fatigue of shoulder ER can appear not only in eccentric contractions to decelerate the arm during throwing motion ${ }^{8}$ but also in concentric action of these muscles during different throwing techniques and throwing direction changes repeated many times during the handball play-match. This suggestion could be supported by previous data of Pontaga and Zidens ${ }^{5}$ who reported that the conventional torque ratio of the ER/IR for male handball players in the middle part of the range of motion (close to 0.80 ) was greater than that in other athletes trained in sports with overhead throwing movements: from 0.55 to $0.68^{1.2}$. These results confirmed that strength increases in both muscle groups were proportional during the training process in handball. Our present and previous results ${ }^{5}$ of shoulder ER/IR torque ratio are very high and in contradiction with the conventional shoulder ER/IR peak torques ratio 0.61 (SD 0.1) and functional ratio 0.68 (SD 0.1 ) in elite male adult handball players obtained by Andrade et al. ${ }^{18}$. These contradictions can be explained by different internal-external rotation planes used in the tests: we used scapular plane but Andrade et al. ${ }^{18}$ frontal plane of isokinetic motions. Greenfield et al. ${ }^{21}$ proved that the shoulder ER strength in scapular plane was significantly higher than those in the frontal plane.

The influence of shoulder IR and ER fatigue resistance on shoulder rotation strength ratios during internal-external rotation movements has not been widely investigated and the results obtained by different Authors are contradictory. Some Authors determined similar fatigue resistance of IR and ER. For example, Mullaney and $\mathrm{McHugh}^{24}$ assessed isokinetic strength endurance of shoulder rotation and reported that IR and ER fatigued similarly during concentric and eccentric contractions. Dale et al. ${ }^{25}$ demonstrated that throwing-related fatigue affected both rotator muscle groups, especially the IR in eccentric contractions appeared due to a throwing protocol of 60 maximal-effort pitches arranged into four innings of 15 pitches per inning in male baseball players. The fa- 
tigue was measured during isokinetic testing that consisted of 12 concentric and eccentric repetitions at a velocity of movement of $300 \%$ for internal and external rotation of the throwing extremity. Andrade et al. ${ }^{18}$ determined that simulated handball game activities (100 steps and 20 arm throws to a goal) caused significant decrease of isokinetic IR and ER peak torques, decrease of $T_{\max }$ of IR was more expressed but the conventional and functional ER/IR peak torque ratios did not changed. The simulated game activities were insufficient to impair the ball throwing performance and are lower in comparison with real intensity of handball play-match loads ${ }^{14,15}$.

Other Authors revealed faster fatigue of ER than IR caused by exercises. For example, Gandhi et al. ${ }^{26}$ determined the voluntary activation failure of the infraspinatus muscle after baseball pitchers developed fatigue from pitching in a simulated game. This was accompanied by lower shoulder ER force and slower pitch velocity. This finding is in agreement with our data obtained on male handball players using isokinetic fatigue protocol, which proved the lower fatigue resistance of the shoulder ER compared to the IR. The same Authors ${ }^{25,26}$ confirmed the negative effect of shoulder muscle fatigue on shoulder joint stability during throwing movements. Chandler et al. ${ }^{27}$ and Ellenbecker and Roetert ${ }^{28}$ found faster fatigue of concentrically activated shoulder ER compared to concentrically activated IR in college tennis players. These Authors suggested that shoulder muscle nonbalanced action increases during prolonged activity, which potentially increases the risk of injury to the athlete throughout the duration of play. This finding confirmed our results of faster ER than IR fatigue due to concentric isokinetic contractions. Our data demonstrated that the fatigue resistance of the shoulder ER was significantly lower compared to the IR fatigue resistance in the dominant and non-dominant arms of semi-professional male handball players. This fatigue resistance difference in IR and ER was a reason for the decrease in the mean $W$ and $P$ ratios of the ER/IR due to repeated internal-external rotation motions in the shoulder joint. Our data agree with the findings of Ebaugh et al. ${ }^{29}$ who revealed that shoulder muscle fatigue due to overhead activities caused less humeral external rotation during arm elevation, and electromyography signs of fatigue were mostly expressed in the infraspinatus and deltoid muscles (ER). These results suggest that the fatigue of shoulder ER during repeated overhead activities appears more rapidly than fatigue of IR.

Clinical relevance: knowledge about shoulder rotation fatigue resistance and ER/IR work and power ratio changes due to repeated overhead activities in handball players would be implicated in shoulder impingement prevention and conservative treatment effect estimation. They are important to elaborate proper training programs for shoulder rotator muscle strength endurance development.

Further research is necessary to determine the effect of real handball play-match activities on isokinetic IR and ER peak torques, the mean work and power, and $\mathrm{ER} / \mathrm{IR}$ ratios of these characteristics.

\section{Conflict of interest}

The Author has no financial or personal relationships with other people or organizations that could inappropriately influence the investigation.

\section{References}

1. Codine P, Bernard PL, Pocholle M, Benaim C, Brun V. Influence of sports discipline on shoulder rotator cuff balance. Med Sci Sports Exerc. 1997;29(11):1400-1405.

2. McMaster W, Long SC, Caiozzo V. Isokinetic torque imbalances in rotator cuff of elite water polo player. Am J Sports Med. 1991;19(1):72-75.

3. Nodehi-Moghadam A, Khaki N, Kharazmi A, Eskandari ZA. Comparative study on shoulder rotational strength, range of motion and proprioception between the throwing athletes and non-athletic persons. Asian J Sports Med. 2013;4(1):34-40.

4. Mikesky AE, Edwards JE, Wigglesworth JK, Kunkel S. Eccentric and concentric strength of the shoulder and arm musculature in collegiate baseball pitchers. Am J Sports Med. 1995;23(5):638-642.

5. Pontaga I, Zidens J. Shoulder invertors and evertors torque production of handball players. J Hum Kinet. 2004;11(8):7582.

6. Pontaga I, Zidens J. Shoulder rotator muscle dynamometry characteristics: side asymmetry and correlations with ballthrowing speed in adolescent handball players. J Hum Kinet. 2014;42(1):41 - 50.

7. Byram IR, Bushnell BD, Dugger K, Charron K, Harrell FE, Noonan T. Preseason shoulder strength measurements in professional baseball pitchers. Identifying players at risk for injury. Am J Sports Med. 2010;38(7):1375-1382.

8. Dillman CJ, Fleisig GS, Andrews JR. Biomechanics of pitching with emphasis upon shoulder kinematics. J Orthop Sports Phys Ther. 1993;18(2):402-408.

9. Chen SK, Simonian PT, Wickiewicz TL, Otis JC, Warren RF. Radiographic evaluation of glenohumeral kinematics: a muscle fatigue model. J Shoulder Elbow Surg. 1999;8(1):49-52.

10. Teyhen DS, Miller JM, Middag TR, Kane EJ. Rotator cuff fatigue and glenohumeral kinematics in participants without shoulder dysfunction. J Athl Train. 2008;43(4):352-358.

11. Joshi M, Thigpen CA, Bunn K, Karas SG, Padua DA. Shoulder external rotation fatigue and scapular muscle activation and kinematics in overhead athletes. J Athl Train. 2011;46(4):349357.

12. Maenhout A, Dhooge F, Van Herzeele M, Palmans T, Cools A. Acromiohumeral distance and 3-dimensional scapular position change after overhead muscle fatigue. J Athl Train. 2015;50(3):281-288.

13. Roy JS, Ma B, MacDermid JC, Woodhouse LJ. Shoulder muscle endurance: the development of a standardized and reliable protocol. Sports Med Arthrosc Rehabil Ther Technol. 2011; $3(1): 1-14$.

14. Michalsik LB, Aagaard P, Madsen K. Locomotion characteristics and match-induced impairments in physical performance in male elite team handball players. Int $\mathrm{J}$ Sports Med. 2013;34(7):590-599.

15. Povoas SCA, Seabra AFT, Ascensao A, Magalhaes J, Soares JMC, Rebelo ANC. Physical and physiological demands of elite team handball. J Strength Cond Res. 2012;26(12):33653375. 
Shoulder external/internal rotation peak torques ratio side-asymmetry, mean work and power ratios balance worsening due to different fatigue resistance of the rotator muscles in male handball players

16. Mullaney MJ, McHugh MP, Donofrio TM, Nicholas SJ. Upper and lower extremity muscle fatigue after a baseball pitching performance. Am J Sports Med. 2005;33(1):108-113.

17. Nocera J, Rubley M, Holcomb W, Guadagnoli M. The effects of repetitive throwing on shoulder proprioception and internal and external rotation strength. J Sport Rehabil. 2006;15(4): 351-362.

18. Andrade MS, Carvalho Koffes F, Benedito-Silva AA, Silva AC, Lira CAB. Effect of fatigue caused by a simulated handball game on ball throwing velocity, shoulder muscle strength and balance ratio: a prospective study. BMC Sports Sci Med Rehabil. 2016;8(13):1-7.

19. Padulo J, Oliva F, Frizziero A, Maffuli N. Muscles, Ligaments and Tendons Journal-Basic principles and recommendations in clinical and field Science Research: 2016 Update. MLTJ. 2016;6(1):1-5.

20. Hadzic V, Ursej E, Kalc M, Dervisevic E. Reproducibility of shoulder short range of motion in isokinetic and isometric strength testing. J Exerc Sci Fit. 2012;10(2):83-89.

21. Greenfield BH, Donatelli R, Wooden MJ, Wilkes J. Isokinetic evaluation of shoulder rotational strength between the plane of scapula and frontal plane. Am J Sports Med. 1990;18(2):124128.

22. Ellenbecker TS, Roetert EP. Testing isokinetic muscular fatigue of shoulder internal and external rotation in elite junior tennis play- ers. J Orthop Sports Phys Ther. 1999;29(5):275-81.

23. Wagner H, Pfusterschmied J, von Duvillard SP, Müller E. Performance and kinematics of various throwing techniques in team-handball. J Sports Sci Med. 2011;10(1):73-80.

24. Mullaney MJ, McHugh MP. Concentric and eccentric muscle fatigue of the shoulder rotators. Int J Sports Med. 2006;27(9): 725-729.

25. Dale RB, Kovaleski JE, Ogletree T, Heitman RJ, Norrell PM. The effects of repetitive overhead throwing on shoulder rotator isokinetic work-fatigue. N Am J Sports Phys Ther. 2007;2(2): 74-80.

26. Gandhi J, ElAttrache NS, Kaufman KR, Hurd WJ. Voluntary activation deficits of the infraspinatus present as a consequence of pitching-induced fatigue. J Shoulder Elbow Surg. 2012;21(5):625-630.

27. Chandler TJ, Kibler WB, Stracener EC, Ziegler AK, Pace B. Shoulder strength, power, and endurance in college tennis players. Am J Sports Med. 1992;20(4):455-458.

28. Ellenbecker TS, Roetert EP. Age specific isokinetic glenohumeral internal and external rotation strength in elite junior tennis players. J Sci Med Sport. 2003;6(1):63-70.

29. Ebaugh DD, McClure PW, Karduna AR. Effects of shoulder muscle fatigue caused by repetitive overhead activities on scapulothoracic and glenohumeral kinematics. J Electromyogr Kinesiol. 2006;16(3):224-235. 